\title{
The Basel NanoBio-Network
}

\author{
Martin Hegner*
}

\begin{abstract}
This article provides a brief overview of the ongoing life science projects within the National Centre of Competence in Research 'Nanoscale Science' (NCCR) network. Within the NCCR, a team of 40 scientists from various research fields is working on the fundamentals of biological nanoscience and on the development of the next generation of nanosensors. Due to the interdisciplinary nature of the projects, the involved Swiss researchers have built during the last three years a network of collaborating experts in the various fields. Such strong networking provides both an efficient approach to research and a good exchange of know-how.
\end{abstract}

Keywords: Bio-sensing · Detection · Diagnostics · Manipulation · Nanoscience · Network · Research coordination $\cdot$ Single molecule $\cdot$ Teaching

\section{NCCR Nanoscale Science}

'Nanoscale Science' as a National Center of Competence in Research (NCCR) is a long-term interdisciplinary research effort focused on nanoscale structures and with the aim to provide new impact and ideas for the life sciences.

Within the NCCR, the University of Basel has the role of a leading house, maintaining a network of universities, federal research institutes and industrial partners in which scientists from a wide variety of disciplines work closely together. The various research groups focus on the following five subject areas:

- Impact of nanoscale science on life sciences and medicine

- Molecular machinery and nanorobotics

- Quantum devices and systems for computing and communication

- Nanoscale science at the ultimate limits

- Nanomaterials ranging from biological systems, carbon-nanotubes to nanoclusters
In the framework of ten projects, various aspects of nanoscale science are being addressed within the above subject areas. The different project teams work at the University of Basel, but some also work at partner institutions within the network. For additional information about the NCCR 'Nanoscale Science' the reader is referred to the internet portal of the NCCR (www.nccr-nano.org).

This paper provides a brief overview of the ongoing life science projects within the NCCR 'Nanoscale Science'. It summarizes a presentation which took place at the BioValley Life Science week (Oct 19 ${ }^{\text {th }}, 2004$ ) at the convention center in Basel. Currently, a team of $\sim 40$ scientists of various research fields (physics, chemistry, molecular biology, microbiology, physical chemistry, medicine, food- and electrical engineering) is working on the fundamentals of biological nanoscience and on the development of the next generation of nanosensors. This note provides a comprehensive but not exhaustive overview of our activities within the life science projects.

\section{The Basel Nano-Network}

Within the ten NCCR projects, two are conducting basic research in the field of life science. The project 'Nanotechnology in Medicine' is headed by U. Aebi while the second project 'Nanotechnology for Cell and Molecular Biology' is headed by A. Engel. Both researchers work at the M.E. Müller Institute of the Biocenter at the University of Basel (www.mih.unibas.ch). These projects involve two kinds of experiments. Firstly experiments on nano-sys- tems involving single biomolecules or small systems including biohybrid nanocontainers which are able to run biochemical and other reactions in a confined space of a few cubic nanometers. The second type of experiment is performed on macro-systems ranging from novel tools for label-free biosensing to nanotools for minimal invasive medical diagnostics and micro-fluidic diagnostic chips (Fig. 1). The applied methods provide the means to manipulate single biomolecules with unprecedented precision and minimal interaction forces. We emphasize that one goal of the NCCR 'Nanoscale Science' is to develop new multifunctional tools for bioanalytics on the nanometer scale.

The current projects are only possible thanks to a fruitful collaboration across research disciplines. One thing we noticed when starting this endeavor is that during the initial setup of the experiments teams composed of researchers originating from different disciplines had to get used to each other. The style in which a physicist pursues experiments can be diametrically opposed from the style that a biologist uses to plan experiments and the vocabulary used for identical issues might be different. In order to overcome such differences and to educate students, the University of Basel has offered for the past two years as the first University in Europe a new curriculum which is entitled 'Nanowissenschaften' (www.nanoscience.unibas.ch/nccr/study).

These new students represent the next generation of researchers who will be able to switch from chemistry to biology or to physics without the barriers of these disciplines. To start the currently ongoing projects, the NCCR required researchers who 


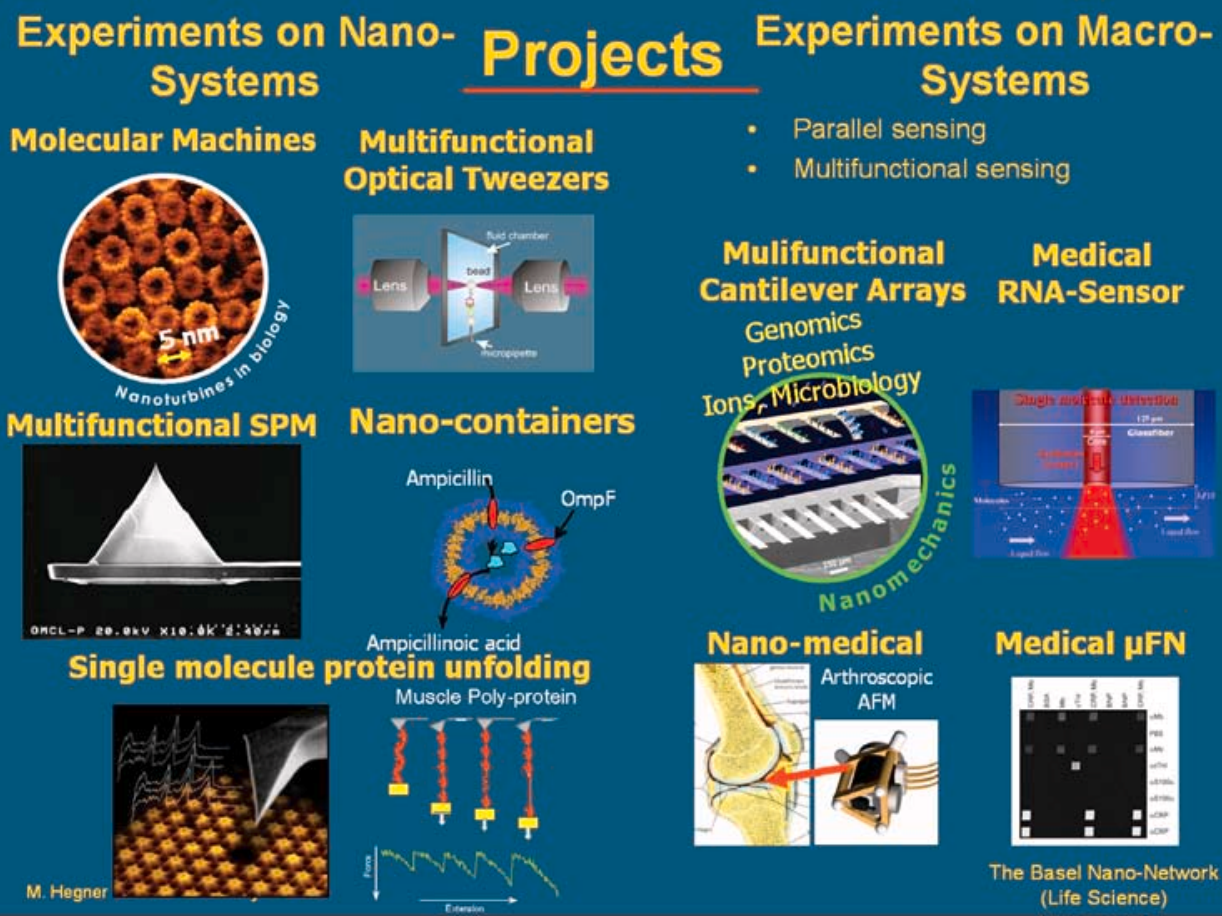

Fig. 1. Overview on the individual life science projects performed within the NCCR Nanoscale Science

came from the traditional disciplines and grouped them within the planned experiments. One fact which greatly facilitates such tasks is that the institutes involved are located in close vicinity to each other. In some of the life science oriented NCCR projects, people from biology, chemistry or physics are interacting with the local industry (Roche, Novartis, etc.). For example, the genomic label-free cantilever array sensors project is a collaboration between the Physics Department (H.P. Lang, C. Gerber, and M. Hegner, http://monet.physik.unibas.ch/ hegner) and the F. Hoffmann-La Roche AG (U. Certa, Roche Centre for Medical Genomics, RCMG, www.roche. com).

The members of the Physics Institute (P. Haas, B. Hecht, M. Calame, H.P. Lang, C. Gerber, and M. Hegner) and the Structural Biology Institute (A. Wild and U. Aebi) develop together with the Departments of Ophthalmology (J. Flammer, www.glaukomforschung.ch), Pathology (M.J. Mihatch, G. Sauter, www.patho.unibas.ch/) and Cardiology (P. Hunziker) of the University Hospital Basel, new miniaturized genomics, proteomics or microorganism biosensors with unprecedented sensitivity (see also www.nano-optics.ch).

A new hand-held label-free nano-mechanical cantilever diagnostics device with selected multiple target-specific synthetic ankyrin repeat proteins as sensors for the rapid diagnosis of multiple pathogens causing febrile illnesses will be developed in collaborative approach involving the Swiss Tropical Institute (P. Beck, M. Tanner, www.sti.ch), the Physics Institute from the University of Basel (C. Gerber and M. Hegner), the Swiss Nano-network partner from the University of Zürich (Biochemistry, A. Plückthun, www.unizh.ch/ pluckth/) and the University of Neuchatel (Institute of Microtechnology, U. Staufer, www-samlab.unine.ch). Such a novel tool could provide the means for comprehensive disease management at the point of care in developing countries. Just from the choice of the project partners it is obvious that without an interdisciplinary approach such a task could never be tackled.

Single biomolecules are monitored by scanning probe microscopy under native conditions in real-time within the groups of A. Engel and U. Aebi. The collaborations with the group of U. Staufer at the University of Neuchatel and the $\mu$-fabrication group at the IBM Research Laboratory cantilevers which not only allow fast imaging but also multi-functionality (e.g. conductivity or nanopipetting in parallel). Such multi-functionality will allow the application of voltage pulses or ion dosages which induce conformational changes in membrane proteins. Since the University of Basel only provides minimal possibility for $\mu$-fabrication, the existing collaboration with the labs in Neuchatel or Zürich enables and opens the way to new exciting research on the nanometer scale in a native environment.

Scanning probe microscopy allows the elasticity of molecules and tissues to be probed. This led to a collaboration of the Zürich (T. Albrecht) provide new combined group of U. Aebi with the group of U.A. Dan Daniels from the Laboratory for Orthopedic Biomechanics at the Felix Platter Hospital in Basel (www.biomechanik.ch). Atomic force microscopy (AFM) was used for measuring cartilage elasticity in physiological buffer environment. The probing of tissues might lead to new medical tools for minimal invasive diagnostics (e.g. in knee joints); this extended collaboration is described in the following section.

Reconstitution of membrane proteins in superstructures formed by amphiphilic block copolymers is one of the research foci of the group of W. Meier at the Physical Chemistry Department (www.chemie.unibas.ch/ meier). The embedding of selected membrane proteins from the group of A. Engel will lead to controlled release devices. Soluble enzymes which are encapsulated can be protected from rougher conditions outside the bio-nanocontainers.

As mentioned earlier some experiments require a covalent attachment of the biomolecules to the interface or chemical modification of the molecule of interest. The chemical expertise of the Organic Chemical Department (B. Giese, $w w w . c h e m i e . u n i b a s . c h / O C)$ and the University of Applied Science of Basel (U. Piles, $w w w . f h b b . c h / c h e m i e)$ allows the exploration and development of new schemes of chemical modification of single molecules which can be investigated by optical tweezers or the chemical interface activation to provide new functional surfaces for biomolecules for new biosensors.

At the Institute of Physics, a new type of combined optical tweezers instrument has been set-up in the group of M. Hegner. The implementation of confocal optical spectroscopy with single photon sensitivity allows force measurement in the sub-piconewton regime combined with parallel optical detection of nanometer distances inbetween single fluorophores (e.g. fluorescence resonance energy transfer) on interacting biomolecules or the detection of local motion on a biomolecule. An overview of the individual experimental projects within the NCCR Nanoscale Science is shown in Fig. 1 and a schematic on the partners interacting locally in Basel is shown in Fig. 2.

\section{The Swiss Nano-Network}

The development of new multifunctional cantilevers and cantilever arrays which are used in many of the life science NCCR projects requires a tight collaboration with institutes that have extended micro- and nanometer fabrication capabilities. The Swiss partners within our network are the University of Neuchatel (Institute of Microtechnology, www-samlab.unine.ch/ 


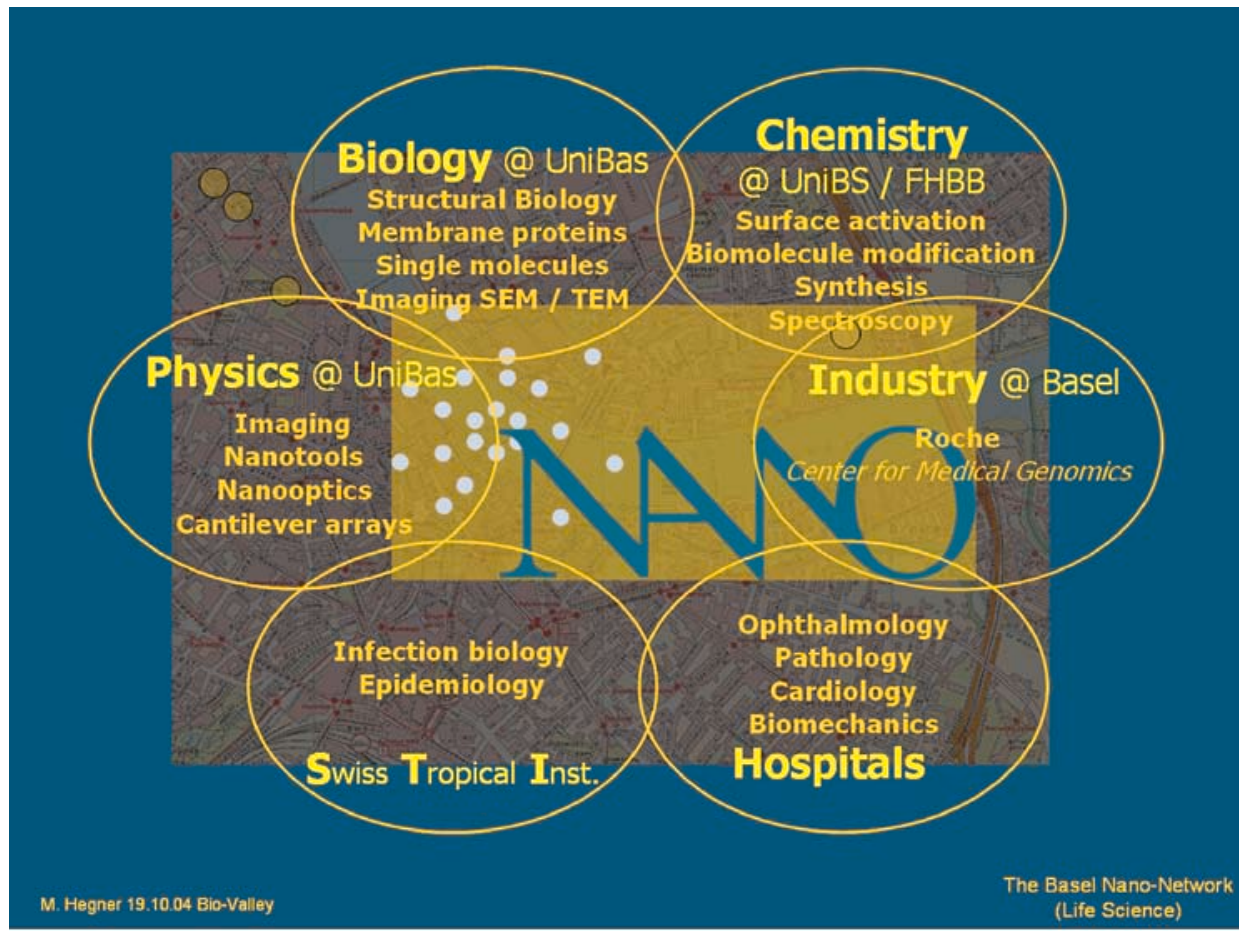

Fig. 2. Overview on the individual partners within the local surroundings of Basel

home.htm) and the IBM Research Laboratory in Zürich (www.zurich.ibm.com) who provide excellent novel $\mu$-fabrication of structures which lead to new tools and allow parallel investigations of multiple functions of single molecules or the label-free biosensing of multiple biomarkers.

Probes are also being developed for medical applications which can measure both temperature and surface structures. A probe of this kind would be able, for instance, to detect initial deposits in arteries and at the same time identify foci of inflammation through temperature measurements. A minimal invasive scanning probe microscopy-based instrument for arthroscopy is under development at the University of Neuchatel (U. Staufer) in collaboration with the group of U. Aebi. Since the size of the device is adjusted to the geometry of common medical arthroscopy instruments, the pathological process in a knee joint could thus be observed and influenced at a much earlier stage than is possible using today's methods.

The researchers from IBM Research Laboratory Zürich (E. Delamarche) and from the Centre Suisse d'Electronique et de Microtechnique (CSEM, H. Heinzelmann, www.csem.ch/) in collaboration with researchers from the University of Basel (P. Hunziker) are developing new medical diagnostics platforms which allow the manipulation of minute nano-liter quantities. Such miniaturization will speed up the time to run the experiments, provide highly sensitive diagnostic tools and reduce costs due to minimum amount of biomolecules used for the assays.
To gain insights into the single molecule mechanics of the most prominent component of the M-band, the modular protein myomesin was expressed in vitro in the group of J.C. Perriard (ETHZ, www.cell.biol.ethz.ch/) and investigated on the molecular scale using various methods (circular dichroism, transmission electron microscopy and SPM). The imaging of the single molecules was performed at the Maurice Müller Institute of the Biocenter (Group of U. Aebi) and the mechanical ma- nipulation at the Institute of Physics by dynamic force spectroscopy in the group of M. Hegner. All these studies revealed that similar to titin, myomesin is a molecular spring characterized by complex viscoelastic properties and might have an influence in specific types of muscle abnormalities. Again, an interdisciplinary collaborative effort was key to the completion of the project. Fig. 3 shows the Swiss collaborating partners within the life science projects of the NCCR Nanoscale Science.

\section{The International Nano-Network}

The launch of the NCCR Nanoscale Science in 2001 created great interest in researchers and universities across borders in Europe and worldwide. Currently, in several European countries Nanocenters have been built and have launched interdisciplinary projects which require a comparable approach to the one started three years ago in Switzerland.

Interdisciplinary nanoscience education across regional borders is promoted by the individual countries. EUCOR Nanoscience for instance is a project to facilitate the mobility of students in the field of nanoscience and nanotechnology in the upper Rhine valley. The current partners of this project are the University of Basel, the University of Freiburg, the University of Karlsruhe, the University of Strasbourg, and the Université de Haute Alsace Mulhouse. The project is embedded in the interregional European organization EUCOR (http://www.eucornanoscience.org/). The regional vicinity of the Universities of the upper Rhine valley

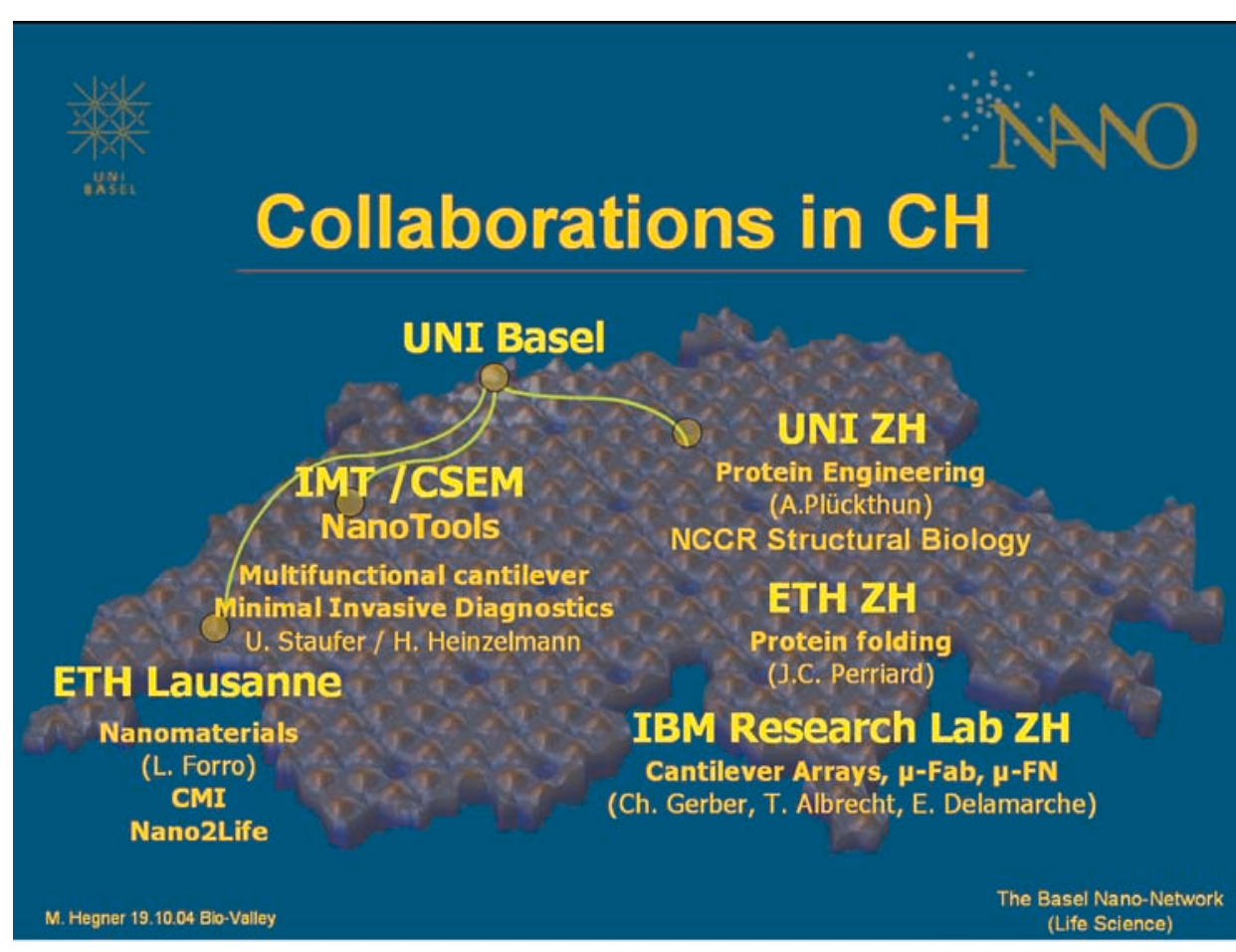

Fig. 3. Overview on the individual collaborating partners in Switzerland 


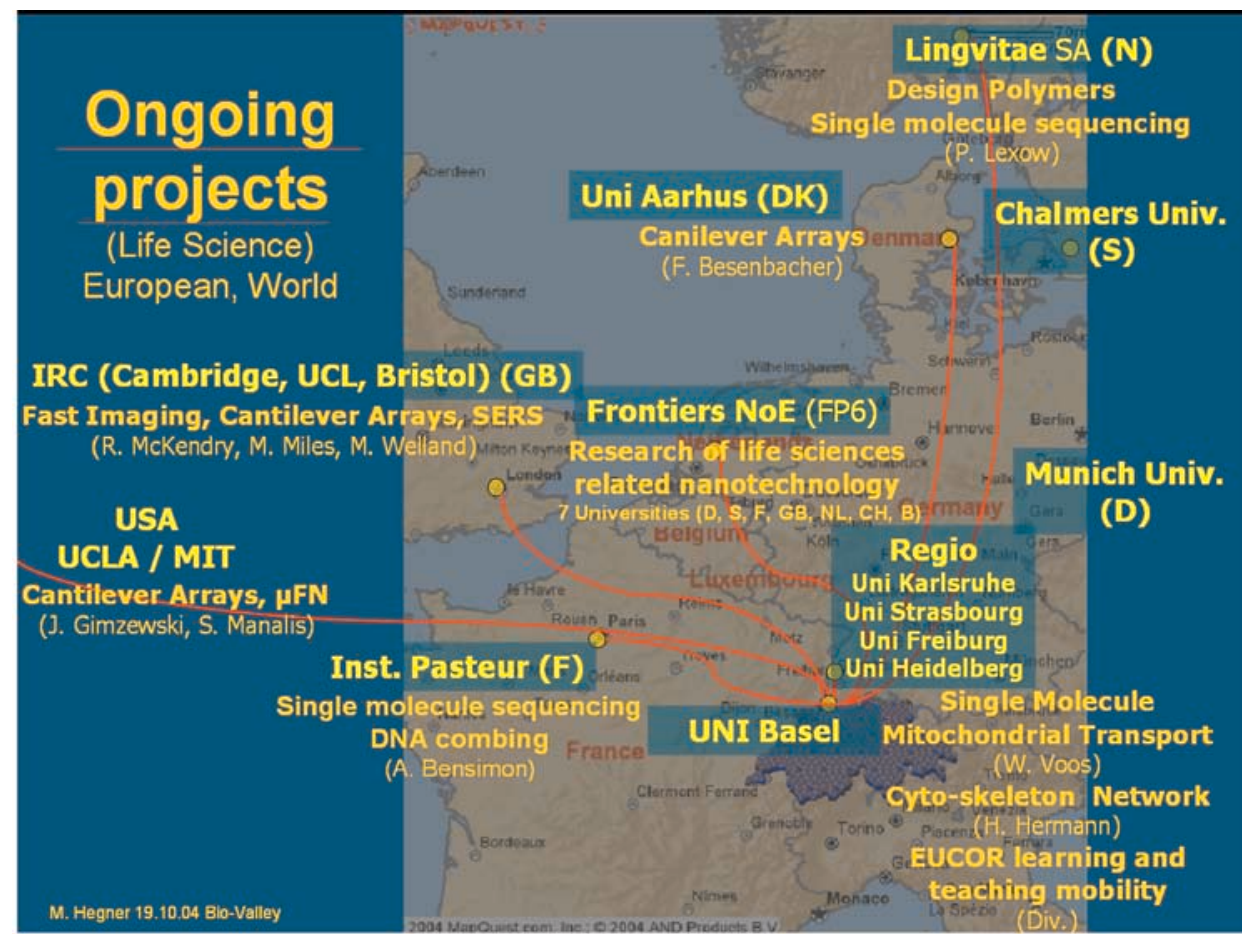

Fig. 4. Overview on the individual programs and partners in Europe and overseas

enables easy traveling and exchange of researchers. Other international life science activities of the NCCR Nanoscale Science in Basel are shown in Fig. 4. A more complete overview on the collaborating partners of NCCR Nanoscale Science shown in Fig. 4 can be gained by following the internet links of the newly launched webpage of the Network of Excellence 'Frontiers' (www.mesaplus.utwente.nl/frontiers/in-

dex.html). This 'Frontiers' network aims at establishing leadership in research and innovation on behalf of life sciences related nanotechnology by creating structures that build on the existing strengths and facilities of the network partners. Currently, most of the partners have ongoing projects with the researchers of the University of Basel. The critical mass of expertise in Frontiers will be obtained by building on existing strength and facilities present at the network partners. Through a joint program of activities (including research activities) the Frontiers network aims at a durable structuring of the way research is carried out in Europe in the area of life sciences related nanoscience.

Received: October 24, 2004 